\title{
BAIANULA EN ISIDORO, ETYMOLOGIAE SIUE ORIGINES, XX, 11, 2.
}

\author{
Juan Francisco MESA SANZ
}

Universidad de Alicante

1. OBJETIVO Y PLANTEAMIENTO DEL PROBLEMA*.

Isidoro, en la exposición de los términos que describen los lechos en lengua latina, presenta uno desconocido hasta ese momento, baianula. La intención con la que abordamos las líneas siguientes no es otra que proponer la traducción del término, así como conocer la realidad designada por este sustantivo. Para llegar a este propósito será necesario acudir a algunas cuestiones en torno a la etimología del término y a la reflexión sobre el autor, la época y el tipo de obra en que localizamos la referencia.

Este autor visigótico hace la siguiente definición de este lecho:

Baianula est lectus qui in itinere baiulatur, a baiulando, id est deportando

(ISIDORO, Etymologiae, XX, 11, 2)

Resulta obvio que podríamos admitir esta definición y concluir parafraseando a Isidoro que es "un lecho de viaje". En efecto es la solución que los diccionarios al uso han adoptado de modo unánime ${ }^{1}$. Sin embargo, al afrontar su estudio ${ }^{2}$ nos asaltaron varios interrogantes: ¿podríamos determinar qué razón ha llevado a Isidoro a emplear este término?; ¿tiene este hecho alguna relación con su etimología? Finalmente y no de menor importancia, ¿que mencione la palabra baianula Isidoro y no aparezca en autores anteriores conecta con el periodo de transición del Mundo Antiguo 
al Medieval? Contestar a estas preguntas supone matizar su contenido semántico y es así que reiteramos nuestro objetivo: establecer la traducción de baianula y la realidad a la que se refiere.

\section{ETIMOLOGIA DE BAIANULA.}

Las palabras latinas que empiezan por la consonante $\mathbf{b}$-se caracterizan por sus problemas de etimología y baianula no iba a ser menos. De hecho, la única mención expresa de ella aparece en un reciente estudio de André (1987) sobre la etimología de palabras 'raras' (sic) ${ }^{3}$ y, en concreto, del término baiulus:

"Mais il n'est pas interdit d'admettre un croisement de Baianus avec gerulus "porteur, portefaix", même si on ne veut pas tenir compte de l'anthroponyme Baius (...) et du baianula "litière de voyage" d'Isidore, Étym. 20,11,2 (var. baianola, badanola), propement "litière à la mode de Baies"."

(ANDRÉ, 1987: 186)

El estudioso francés no le presta mayor atención a este término. Toda su argumentación se encamina a justificar la relación entre baiulus y Baiae. Esta conexión es defendida por medio de comparaciones con la situación actual en la lengua francesa, así como la utilización de algunos términos muy concretos de la lengua latina: de la misma manera que Indus (propiamente 'hindú') pasó a ser considerado 'conductor de elefantes'4, baiulus ('habitante de Bayas') evolucionaría a 'porteador'. No es este el lugar para discutir sus tesis sobre el origen de baiulus, aunque podemos apuntar su carácter escaso. En el asunto que nos preocupa, la palabra baianula, no esclarece nada en absoluto. Admite sin ninguna argumentación el origen geográfico del objeto descrito $y$, por tanto, de la denominación. Sin embargo, el mismo desarrollo teórico de la etimología de baiulus brinda una interpretación diferente de la apuntada. Nos referimos a la presentación de la palabra liburna:

Liburnus, nom d'un peuple dalmate de pirates fournissant des esclaves, désigne soit des appariteurs dans les cours de justice (Mart. 1, 49, 33 5 ; Juv. 4, 75), soit un porteur de litière: Juv. 3, 239, liburna "litière liburnienne", i.e. "portée par des Liburniens" 6 , cf. C.G.L. 5, 656, 6, Liburni id est homines lecticarii uel praecones.

(ANDRÉ, 1987: 186)

Si admitimos la relación entre baianula y baiulus que sugiere este autor y consideramos que liburna es 'una litera transportada por liburnos', concluiremos que baianula es una 'litera transportada por baiuli'. Ya hemos dicho que no resulta más explícito, ya que no se dedica al origen de esta 
palabra que, a partir de la afirmación que realiza, podemos deducir que le parece de etimología clara.

En oposición a esa claridad nosotros acabamos de apuntar dos posibilidades distintas: por un lado, la procedencia señalada del topónimo Baiae, pero, por el otro, la asociación de baianula con baiulus. Incluso nos atrevemos a postular una tercera que aunaría las dos anteriores: baianula procedería de Baiae a través de su relación con baiulus, ya que André afirma que Baiae es el étimo de éste.

La primera posibilidad, la procedencia directa del topónimo, justifica la traducción e interpretación de la realidad que sugiere André ('litera a la moda de Bayas'). Asimismo tal afirmación resulta de una gran claridad morfológica: del topónimo se forma el gentilicio Baianus y, posteriormente, baianula, por medio de la adición del sufijo -ulus. En este último paso (o quizá en el anterior) se registraría una sustantivación del término, ya que partimos de una base adjetival que, aunque frecuente en los alargamientos y sufijaciones, debe tener una justificación. Por supuesto es suficiente tratar de referirse a una realidad nueva; sin embargo, nos interesa conocer en qué momento se pudo producir su aparición. Es conocido, ya que así lo manifiestan los testimonios literarios, que a fines del periodo republicano, y mucho más en el imperial, el establecimiento termal de Bayas se convirtió en el lugar predilecto de la aristocracia romana; sin duda, es este arco temporal que va del siglo I a.C. hasta fines del siglo II d.C. el que posee más probabilidades de haber contemplado el nacimiento de un término relativo al contenido apuntado por André, 'moda de Bayas'. Con anterioridad a esa fecha el emplazamiento de Baiae carecía de importancia; posteriormente, ya en la crisis del siglo III d.C., deja de tenerla, de tal manera que no parece posible que se pudiera originar 'una moda' después de esa fecha. Al practicar el análisis de baianula, observamos que la base sobre la que se forma es el gentilicio Baianus, el cual es atestiguado por vez primera en la República tardía ${ }^{7}$, cuando, como hemos dicho, empieza a popularizarse esta zona entre los miembros de las clases superiores romanas. La afición a las aguas termales de Bayas permitiría suponer que una litera de unas características que desconocemos se prodigó en aquella zona y se extendió con posterioridad a otros lugares. En la formación del nombre se produciría un simple proceso de elipsis: "Baiana lectica > ${ }^{*}$ baiana; posteriormente se realizaría la adición de un nuevo sufijo con lo que se culminaría el proceso de formación 8 . Hasta aquí una argumentación que no podemos basar en testimonios dada su inexistencia, pero que discutiremos en los párrafos siguientes.

Después de que hemos dejado abierta la solución de la primera posibilidad etimológica, acudimos a la segunda: baianula procede de baiulus. 
Con anterioridad nos hemos referido a esta relación; afirmábamos que, de la misma manera que liburna era considerada (o, mejor dicho, procedía) de liburnus, de tal modo que significaba originariamente 'litera transportada por liburni', baianula podía asociarse a baiulus y referirse a algún tipo de 'litera transportada por baiuli. En este apartado nos asalta una duda: ya que es muy clara la raíz que aparece en el sustantivo, baiul-, a partir de la que se forma, por ejemplo, el verbo baiulare, hubiese sido más lógico esperar una forma *baiulona y no baianula; esta última enmascara la raíz. Ante este problema sólo concebimos dos vías de solución: (i) considerar que baiulus y baianula poseen orígenes diferentes; o (ii) admitir la tesis de André por la cual baiulus procede de Baiae. La segunda de las posibilidades es la que nos conduce a la tercera que pretendíamos analizar, en la cual baianula se relacionaba con Baiae a través de baiulus; algo que podríamos considerar como una hibridación de las dos soluciones anteriores.

La tercera propuesta supone que la realidad mencionada debería ser 'una litera transportada por baiul' en primer lugar; en segundo lugar, el momento en el que se originó esta palabra nos debería dar muestras de que en la conciencia de los usuarios de la lengua baiulus se asociaba de forma inequívoca con el asentamiento geográfico de Baiae. Dicho de otro modo, los textos latinos en los cuales aparezca la referencia deben mostrarnos que en la utilización del término se percibía la formación del compuesto *baia-ulus $>$ baiulus, pese a que, con posterioridad, pasara a referirse a una realidad distinta. Si descubrimos que se mantiene el carácter motivado del compuesto, es posible que el hablante reconstruyera la raíz para la formación de otro, lo que haría innecesario hipotetizar la existencia de *baiulona. La valoración de este hecho la buscaremos en dos momentos diferentes: por una lado, en los testimonios más antiguos del uso de baiulus y baiolare, puesto que constituyen los hitos temporales teóricamente más cercanos al origen del vocablo y, por el otro, los datos que nos ofrecen los autores republicanos y altoimperiales, ya que en su época habíamos afirmado que pudo originarse el término con el significado de 'a la moda de Bayas'.

Las noticias históricas no interponen veto alguno a tal relación, puesto que no es necesario pensar que los habitantes de las aquae Cumanae se desplazasen a Roma. La bahía de Nápoles fue por excelencia la zona portuaria de Roma hasta que, ya en época de Claudio y muy especialmente con Trajano, se acometieron los grandes trabajos de acondicionamiento del puerto de Ostia para que pudieran llegar hasta él los enormes buques de grano 9 que suministraban a la Urbe. Putéolos era el puerto principal y no es descabellado pensar que los habitantes de la pequeña localidad cercana se aproximasen hasta él para trabajar en las labores de descarga y transporte ${ }^{10}$. Los testimonios arqueológicos 
muestran la existencia temprana de pobladores en toda la bahía y especialmente de la que luego será el gran centro de recreo de la aristocracia romana ${ }^{11}$. A esta argumentación se añaden los usos del sufijo - ulus ${ }^{12}$. Si admitimos la argumentación de André observamos la formación de dos étnicos diferentes a partir de un topónimo: Lugdunensis, 'habitante de Lugdunum' frente a Lugdunolus, apelativo (¿cariñoso o despectivo?) de un soldado que se afinca en Africa ${ }^{13}$; y Baianus, 'habitante de Baiae' frente a baiulus, '¿habitante de Baiae? dedicado al oficio de porteador (oficio denigrante)'. Dicho de otro modo, junto a la formación de un gentilicio a partir del topónimo, estas dos palabras parecen señalar que el sufijo - ulus se está destinando a la formación de un antropónimo de tono jocoso (no creemos que fuera otra la intención en el caso del legionario) o despectivo, contenidos ambos reconocidos en el valor diminutivo que originariamente posee. No obstante, cuando registramos las apariciones más antiguas de los términos baiulus y baiolare el hablante no reconoce ya la formación del derivado, si bien mantiene su tono despectivo. Los textos plautinos son una buena muestra de este carácter inmotivado ${ }^{14}$ :

1) PLAUTO, Asinaria 660: En la escena se encuentran el esclavo Leónidas y el amito Argiripo; juegan con el hecho de cargar con un saco lleno de dinero:

LE. nolo ego te, qui erus sis, mihi onus istuc sustinere.

ARG. quin tu laborare liberas te atque istam imponis me?

LE. ego baiiolabo ${ }^{15}$, tu, ut decet dominum, ante me ito inanis.

2) ID., Mercator, 508: La conversación se realiza entre el anciano Lysímaco y la prostituta Pasicompsa; el primero intenta comprarla y ésta realiza una enumeración de tareas que considera denigrantes y no realiza como prostituta:

PA. namque edepol equidem, mi senex, non didici baiiolare nec pecua ruri pascere nec pueros nutricare.

3) ID., Poenulus, 1301: En un largo parlamento al inicio de la escena Antamoenides considera que si una joven abraza a un baiulus en público es una acción denigrante:

et ea est certo. iam pridem ego me sensi nihili pendier.

non pudet puellam amplexari baiiolum in media uia?

iam hercle ego illunc excruciandum totum carnufuci dabo.

sane genus hoc mulierosumst tunicis demissiciis.

4) ID., ibid., 1354: Estamos en la penúltima escena y ya en el desenlace final. Véase de nuevo el juego y el contenido peyorativo del término:

LY. de prandio tu dicis. debetur, dabo.

AG. duplum pro furto mi opus est. LY. sume hinc quid lubet. 
HA. et mihi suppliciis multis. LY. sume hinc quid lubet.

AN. et mihi quidem mina argenti. LY, sume hinc quid lubet. collo rem soluam iam omnibus quasi baijolus.

En suma, los ejemplos que nos suministra Plauto dan cuenta de un contenido netamente peyorativo, pero en absoluto de una asociación geográfica. Podría argumentarse en contra que ese mismo contenido despectivo es el que reconocíamos en el sufijo -ulus; cierto, mas no lo es menos que el contexto en el que se introducen los términos analizados subrayan que tal carácter peyorativo procede del oficio designado y no del sufijo que se emplea. En otras palabras, no se está produciendo un fenómeno como el de la palabra española 'hombrecillo', sino más bien el uso de 'esclavo' en una oración como 'trabajo como un esclavo', en la que el hablante jamás piensa (ni reconoce) el origen de este término a partir del gentilicio 'eslavo'. Así, pues, creemos que ya desde su origen, aun admitiendo el establecido por André, baiulus fue considerado un compuesto inmotivado, utilizado para la descripción de un trabajo y no como diminutivo despectivo de un gentilicio. Por ello, no creemos que el periodo arcaico sea el momento en el que se origina nuestra palabra, ya que el hablante latino raramente habría reconocido en baiulus y baiulare la raíz baia- que forma la base de ésta. Deberíamos considerar el origen independiente para cada vocablo y acudir en el caso de baianula a la cronología que hemos manifestado más arriba.

Los textos que nos ofrecen el uso de estas palabras y se corresponden con el arco temporal en el que situábamos el posible origen de nuestro término, si atendíamos a la definición de André, no determinan un cambio sustancial. Baianula no está representado; si, en cambio, baiulus. Cicerón mantiene el carácter despectivo; así, por ejemplo, en de oratore II, $40^{16}$ :

remigem aliquem aut baiulum: nam hesterno sermone unius cuiusdam, "operis" ut ait Caecilius "remigem aliquem aut baiulum" nobis oratorem descripseras, inopem quendam humanitatis atque inurbanum.

Nuestra reflexión se repite: no hay ninguna referencia a un contexto geográfico que justifique la asociación de los términos. Es más, el final del siglo II d.C. nos trae los primeros cambios en la concepción en la que se tiene a los trabajadores designados bajo este vocablo. Ya Apuleyo utiliza el término baiuli sin carácter despectivo cuando menciona las campañas de Alejandro (Florida VII, p.27) y en un texto religioso denomina de este modo a quienes transportan las enseñas sagradas (mund. 34): Inerant boni baiuli, religiosis sarcinis occupati. Se ha iniciado un interesantísimo proceso semántico que conduce a baiulus de ser un denigrante 'porteador' a un alto 
cargo de la administración y de la Iglesia ${ }^{17}$. En suma, lejos de aproximarse a un supuesto origen geográfico, baiulus y sus compuestos desarrollan todo un proceso diacrónico que merece ser estudiado con más detalle; éste, a su vez, imposibilita cualquier relación etimológica baianula < Baiae mediada por baiulus.

Concluimos así este apartado que nos muestra tres vías posibles para enfrentarnos al estudio del texto de Isidoro. La primera, expresada por André, que asocia baianula directamente con Baiae y que nosotros hemos ubicado en un marco temporal muy concreto (del siglo I a.C. al siglo II d.C.); la segunda en la que hemos postulado su relación con baiulus, si bien creemos que hubiese sido esperable la forma *baiulona; finalmente, la tercera relaciona las dos anteriores a partir de la etimología Baiae que André establece para baiulus. Las dos primeras han quedado abiertas debido a la carencia de testimonios que nos conduzcan a elegir una; la última ha sido desechada porque los testimonios más antiguos de baiulus, así como los correspondientes al lapso temporal indicado, no reflejan un posible origen geográfico, de tal manera que difícilmente podría dar lugar a un nuevo compuesto a partir de una supuesta base etimológica *baia-. En pocas palabras, nos acercamos al texto de las etimologías con dos propuestas en cuanto a su contenido: 'litera a la moda de Bayas' o 'litera transportada por baiuli?.

\section{BAIANULA EN ISIDORO, ETYMOLOGIAE, XX, 11, 2.}

La mención de la palabra la encontramos en medio de la exposición de las diferentes denominaciones de los lechos. Nos vamos a permitir reflejar a continuación el texto completo ${ }^{18}$ :

1. Lecticae a lectis herbis vocatae. Stratus ab sternendo dictus, quasi storiatus. In his solis antiqui ad dormiendum adcubabant, nondum laneis stramentis repertis. Storia, quod sit terra strata. 2. Cama est brevis et circa terram; Graeci enim camaiv breve dicunt. Cubile est cubandi locus. Grabatum graecum est. Baianula est lectus qui in itinere baiolatur, a baiolando, id est deportando. 3. Pulvinar lectus divitum est: inde et pulvillus. Spingae sunt in quibus sunt spingatae effigies, quos nos gryphos dicimus. Punicani lecti parvi et humiles primum a Cartagine advecti, et inde nominati. 4. Lecticae, sive plutei lecti. De quo Rutilius Rufus de vita sua (13): 'Primum', inquit, 'contra consuetudinem imperatorum ipse pro lectis lecticis utebatur.'

Los cuatro párrafos que hemos transcrito aquí nos muestran un cierto principio organizador en la exposición de Isidoro. El primer párrafo nos presenta las denominaciones empleadas para describir los lechos apoyados 
en el suelo, o la referencia utilizada para 'dormir en tierra'; el tercero nos ofrece lechos con características muy concretas, 'de los dioses', 'con esfinges', 'procedente de Cartago'; el cuarto se destina en exclusiva a lectica. Este último le supone un esfuerzo, puesto que lectica es el término clásico para 'litera de viaje' y en el periodo medieval mantendrá este significado; sin embargo, Isidoro ha destinado el termino baianula a su expresión. Es este párrafo, el segundo, sorprendente puesto que contiene dos términos que registramos por primera vez, cama y baianula, junto a otros dos con pocas referencias a un lecho: uno por su escasa aparición (casi siempre en textos cristianos), grabatum, y el otro por ser ésta una acepción marginal, cubile. ¿Cuál es la realidad que quiere encorsetar Isidoro en este apartado? Es el momento de acudir a los antecedentes que expresábamos en el estudio de la etimología.

En cuanto a la adscripción geográfica que nos anunciaba André, cuesta pensar que no la hubiese empleado aquí en caso de considerarla prioritaria; así lo hace, por ejemplo, con los Punicani que, por cierto, también conocemos de modo exclusivo por medio de este autor. Es más, podemos observar que Baiae no tenía ninguna relación con baianula y baiolare en la mente de Isidoro, si atendemos a la mención de baia y Baiae en la misma obra:

- baia: XIV, 8, 40. Portus autem locus est ab accesu ventorum remotus, ubi hiberna opponere solent: et portus dictus a deportandis commerciis. Hunc veteres a baiolandis mercibus baias vocabant, illa declinatione a baia baias, ut a familia familias.

- Baiae: (Cuando realiza la descripción de Italia) XIV, 4, 19. Habet lacus Benacum, Avernum atque Lucrinum; fluvios Eridanum et Tiberim; et tepentes fontibus Baias.

Observamos que considera dos palabras completamente diferenciadas baia, 'puerto' y el topónimo Baiae que debe su nombre a la presencia de aguas termales. Incluso el hecho de que la primera sea fruto de una mala lectura de Servio casi con toda seguridad ${ }^{19}$ abona con más fuerza esta disociación entre las raíces de los términos. Finalmente, Isidoro no podía pensar en un término que se relacionase con una localización geográfica que hacía varios siglos que había perdido toda importancia. En consecuencia, creemos que debe desecharse la definición de André, no desde el punto de vista etimológico (siempre discutible), sino desde el punto de vista semántico: ya que no existe indicio alguno que nos permita admitir que la realidad referida por Isidoro pueda remitirse a la localidad de Bayas. 
La segunda posibilidad asociaba este término con baiulus. Esta es la solución hacia la que apunta el propio texto de las Etimologías, ya que para su definición se vale del correspondiente compuesto verbal, baiolare. Ahora bien, decíamos antes que en tal caso la forma esperada hubiese sido *baiulona y no baianula. En este sentido la mención anterior de la palabra baia asociada al mismo verbo nos muestra que para Isidoro el origen es el mismo; incluso creemos que ha podido producirse un cruce en la exposición de este autor. Todo ello, claro está, siempre y cuando admitamos baianula como la lectura correcta que debe figurar en la edición crítica, aspecto no del todo claro, puesto que los manuscritos nos ofrecen lecturas diferentes: baianola en los códices Bernensis y Toletanus (nunc Matritensis), badanola en el Karolinus, baionula; incluso esta palabra, desconocida en los textos clásicos, aparece en los textos medievales en gran medida por imitación del texto isidoriano y Du Cange ha incluido las diferentes variantes bajo el lema bajulona, ya que tal fue la corrección que aplicaron algunos glosógrafos medievales 20 . En resumidas cuentas, ya sea porque se ha producido una equivocación por parte de Isidoro en su afán clarificador del origen de las palabras, ya porque nos encontramos ante un problema de crítica textual, la realidad referida por Isidoro se asocia a baiulare y, por tanto, a baiulus y no, es obvio, a Baiae. En concreto, sería una 'litera trasportada por medio de porteadores'.

Hasta este punto hemos abordado el análisis de la referencia a partir de los testimonios que nos ofrece el Mundo Antiguo, sin embargo, ya hemos recurrido en dos ocasiones a la época de la redacción de las Etimologías: para desechar la pujanza de Bayas y para localizar bajulona en los textos medievales. $Y$ es que Isidoro, como apunta Cremascoli21, nos presenta "plus qu'un résultat, (...) un procès en cours, et le doit être entendue comme "practique, active et productive, d'une intelligence en plein effort"." Este autor es igualmente el modelo de la glosografía medieval en la que observamos que se adopta toda palabra, las que se habían utilizado y las que eran de uso, y, por ello, también pudieron ser incluidas las corruptas y fantásticas ${ }^{22}$. Baianula, por la vigencia posterior, así como la aparición de sinónimos como baiulatoria, no se debe incluir en las últimas; no es de ningún modo una ficción intelectual. Sí corrupta en la medida en que hemos apuntado las lecturas diferentes que nos ofrecen los manuscritos e incluso la corrección de la glosografía posterior para introducir el término bajulona. Finalmente, el hecho de que no conozcamos ningún texto que contenga este vocablo con anterioridad a Isidoro, nos lleva a afirmar que este autor quiere explicar un término de su entorno; es lo mismo que ocurre sin ir más lejos con cama, cuya aparición posterior en nuestra lengua convierte en ociosa cualquier explicación. En suma, desea explicar un término del 
momento en el que escribe y a ese mundo visigótico de los siglos VI y VII d.C. le debemos dirigir nuestras preguntas.

Las Etimologías no reflejan nunca el término baiulus. Así la relación de este lecho se establece en exclusiva con el verbo baiolare, 'transportar'; de acuerdo con ello, la referencia sería a un 'lecho transportable' sin más indicaciones que ese carácter de in itinere, 'de viaje', que ofrece el propio autor. No obstante, no podemos concluir nuestra exposición en este punto. A lo largo de nuestro artículo, hemos expuesto el sustantivo, baiulus, y el verbo, baiolare, formando unidad; ahora la hemos disuelto. Lo hemos hecho en primer lugar por la falta de una mención expresa del primero, pero también porque éste había iniciado una evolución que le llevaría a los medievales balius, balivus, baillivus de los cuales proceden bailiff y baile 23 . Apuntábamos que en el siglo II d.C. registrábamos los primeros cambios en el contenido semántico; en el siglo III d.C. localizamos una inscripción en Germania con referencia a un vexillarius (relacionado con el culto a la diosa Victoria) ${ }^{24}$, en la que baiolus se identifica con el cargo de 'correo oficial'; otra inscripción funeral cristiana de cronología incierta, pero posterior al III y anterior al VI, muestra este término en función de cognomen del sirviente de un pontifex ${ }^{25}$; finalmente, Simaco, en el final del siglo IV, los menciona como los frugis et olei baiuli (Epistulae $X, 27$ ) refiriéndose a ellos como una corporación equivalente a los saccarii, lo que, si no se trata de una simple variación literaria, manifiesta esa pérdida del carácter peyorativo y el que deban considerarse dotados de mayor estatus, puesto que se habían hecho con el monopolio de las labores de carga y descarga de las mercancias del puerto (Ley de Valentiniano I (364): Cod. Theod. XIV, 22, 1) ${ }^{26}$. Ya hemos mencionado igualmente que en el periodo medieval el término es utilizado para referirse a altos cargos de la administración y de la Iglesia. Estos elementos de juicio nos indican que la denominación 'litera transportada por baiuli no se corresponde con la realidad en el momento en que Isidoro redacta su obra. ¿Es baianula la "litera de viaje de un baiulus'? Es este un interrogante que queda abierto y cuya solución sólo se podrá apuntar tras el análisis de la evolución semántica de baiulus y sus compuestos desde su origen hasta el periodo medieval, antes y después de Isidoro, para conocer cuál era la concepción expresada por estos términos en el momento de la redacción de las Etimologías.

\section{CONCLUSION Y EPILOGO.}

El estudio que acabamos de realizar del pasaje de Isidoro, en el que se registra la presencia del término baianula, nos ha llevado a desechar la posibilidad, apuntada por André, de que este vocablo describiese propiamente 'una litera a la moda de Bayas'. Frente a ello, la descripción 
adecuada parte de la hipótesis por la cual baiulus (baiolus), baiolare y baianula son miembros de una misma familia etimológica; al margen de su origen de difícil solución, los testimonios conservados nos han mostrado que, desde el primer momento, se ha perdido (en caso de haber existido) toda referencia geográfica a la localidad de Bayas e igualmente tal asociación resulta poco probable en la época en que redacta Isidoro.

La reflexión anterior conduce a que el término esperable es baiulona y así nos aparece en la glosografía posterior. El pasaje de las Etimologías no está exento de problemas textuales, sin embargo, creemos que la forma que utilizó su autor fue baianula, ya que procede de su propia argumentación: baia es una denominación de puerto que procede de baiolare, por tanto baianula procede de baiolare.

Establecidas las premisas anteriores la realidad referida es 'lecho de viaje, litera'. Ahora bien, nuestra propuesta a raíz de las conclusiones que obteníamos de los textos clásicos anunciaba un 'lecho transportado por baiuli, solución que no se corresponde con la ahora adoptada. Esto es debido a que en nuestro trabajo hemos registrado una interesante evolución en el contenido expresado por baiulus; a su vez, ese contenido abre un nuevo interrogante que sólo podrá cerrarse en el futuro con un estudio completo del término baiulus y sus derivados.

\section{BIBLIOGRAFIA:}

AMSLER, Mark, Etymology and Grammatical Discourse in Late Antiquity and the Early Middle Ages, Amsterdam, Philadelphia, John Benjamins, 1989.

ANDRÉ, Jacques, 1987 "Étymologies et mots rares", Revue de Philologie 61, pp. 185-192.

Apuleius, Opera omnia, ed. G. F. Hildebrand, Hildesheim, Olms, 1968 (=Leipzig, 1842).

Astin, A.E. - Walbank, F.B.A. - Frederiksen, M.W. - Ogilvie, R.M., The Cambridge Ancient History, VIII: Rome and the Mediterranean to 133 B.C., Cambridge, Cambridge University Press, $1989^{2}$.

CHuRCHILL SEMPLE, Ellen, The geography of the Mediterranean region (its relation to ancient history), London, Constable, 1932.

CIL: Corpus Inscriptionum Latinarum.

Cremascoli, Giuseppe, "Note sur des problèmes de lexicographie médiévale", en CALBOLI, G (ed.), Latin vulgaire - Latin tardif / (Actes du llème. Colloque international sur le latin vulgaire et tardif (Bologne, 19 août2 september 1988), Tübingen, Max Niemeyer, 1990, pp. 75-88

Du CANGE, Domino, Glossarium mediae et infimae Latinitatis, Niort, L. Favre, 1883.

Ernout, A. - Meillet, A., Dictionnaire étymologique de la langue latine (Histoire des mots), Paris, Kliencksieck, $1960^{4}$. 
FRUYT, Michèle, "Étude sémantique des diminutifs latins. Les suffixes -ulus, culus, -ellus, -illus... Dé-substantivaux et dé-adjectivaux", en LAVENCY, M. LongréE, D. (eds.), Actes du Veme. Colloque de linguistique latine, Louvain-La-Neuve/Borzée, 31 mars - 4 avril 1989, Louvain, Peeters, 1989, pp. $127-138$.

FRUYT, Michèle, Problèmes méthodologiques de dérivation à propos des suffixes latins en ...cus, Paris, Klincksieck/Publications de La Sorbonne, 1986

GAFFIOT, F., Dictionnaire Latin-Français, Paris, Hachette, 1934.

GAIDE, F., "Formes élargies' du 'latin vulgaire': un cas trés particulier de la dérivation", Latomus 47 (1988), 584-592.

ICUR: De Rossı, Joannes Baptista - FERruA, Antonius, Inscriptiones Christianae Vrbis Romae septimo saeculo antiquiores, VI, Vaticano, Pont. Institutum Archaeologiae Christianae, 1925.

ISIDORUS, Etymologiarum sive originum libri $x x$, ed. W.M. LINDSAY, Oxford, 1911.

JUVENAL, Sàtires, ed. y trad. cat. Manuel BALASCH, Barcelona,Fundació Bernat Metge, 1961.

Kajanto, I., The Latin Cognomina, Roma, Bretschneider, 1982 (=Helsinki, 1965).

LeUmanN, Manu, Lateinische Laut- und Formenlehre, München, C. H. Beck, 1963.

Niefmeyer, J.F., Mediae Latinitatis Lexicon Minus, Leiden, E. J. Brill, 1976.

NISSEN, Heinrich, Italische Landeskunde, 2 vols., Berlin, Weidmann, 1902.

PAVIS D'EscuRAC, Henriette, La préfecture de l'annone, service administratif impérial d'Auguste à Constantin, Roma, École Française de Rome, 1976

PRINZ, Otto, "Mittellateinische Wortneubildungen, ihre Entwicklungstendenzen und ihre TriebKräfte", Philologus 122 (1978), pp. 249-275.

TLL: Thesaurus Linguae Latinae, Leipzig, Teubner, desde 1900.

TUCKER, T.G., A concise etymological dictionary of latin, Halle, Max Niemeyer, 1931.

WALDE, A., 1938, Lateinisches Etymologisches Wörterbuch, Carl Winter, Heidelberg. 


\section{NOTAS}

* El presente trabajo se ha beneficiado de una ayuda de la Conselleria de Educación i Ciencia de la Generalitat Valenciana para estancias en el extranjero.

1 Así, por ejemplo, GAFFIOT (1934: s.v. bajonola (-nula)), o T.L.L. (s.v.).

2 Con motivo del Proyecto de Investigación 'Diccionario Latino-Español" (CICYT, PB90-0525-C02-01).

3 El subrayado es nuestro.

4 Livio, Ab urbe condita, XXXVIII, 14, 2.

5 Marcial, Epigrammata, I, 49, 33: procul horridus Liburnus et querulus cliens, / imperia uiduarum procul.

6 La referencia corresponde realmente al verso 240: (...) et ingenti curret super ora Liburna /(...). El subrayado es nuestro.

7 El testimonio más antiguo corresponde a Cicerón, Att. 14, 8, 2.

8 Podría incluso pensarse que nos hallamos ante un alargamiento propio del latín 'vulgar' (GAIDE, 1988: 586) o 'tardío', lo que nos ayudaría a matizar las fechas; veremos que no es necesario.

9 Pavis D'Escurac, 1976: 102-3: "Avant l'aménagement de son port, Ostie n'eut qu'un rôle réduit dans le trafic annonaire; Pouzzoles était le grand port importateur de grain. Même après les travaux de Claude à Ostie, Pouzzoles conserva une activité annonaire considérable; ce n'est qu'à partir du creusement du Portus de Trajan que Puteoli commença à souffrir vraiment des effects de la concurrence."

10 Durante todo el siglo II a.e. las ciudades de Ostia, Puteolos y Pompeia crecen como resultado del movimiento de población hacia las ciudades, en especial hacia los grandes establecimientos portuarios (Astin WALbanK - FRederiKSEN - OGILVIE, 1989: 219 y 436).

11 La actual bahía de Nápoles fue siempre un lugar enormemente atractivo para el comercio marítimo; así localizamos las ciudades de Cumae y Neapolis, o el puerto de Puteolos. Si atendemos a la referencia de Livio $(\mathrm{XLI}, 16)$ y Lucrecio (VI, 747), las fuentes termales (Baiae, aquae Cumanae) eran conocidas por lo menos desde 176 a.e. Es más, conocemos por Horacio $(E p .1,15,7)$ unas Baiae ueteres diferenciadas de lo que se convertirá en lugar de ocio y recreo de la aristocracia (NISSEN, 1902: II, 733-2). Cf. para más referencias CHURCHiLL, 1932: 51.

12 Simple diminutivo empleado en la formación de compuestos (LEUMANN, 1963: 223), asociado a la formación de cognomina diminutivos, despectivos, cariñosos (KAJANTO, 1982: 123) o con todos esos significados (LEUMANN, 1963: 305). En el campo semántico de los lechos lo encontramos en lectulus y lecticula, habiéndose formado también con diminutivo lectica (FrUYT, 1989: 143-4). CIL VIII, 27850 (cf. KAJANTO, 1982: 203) 
14 FruYT, 1989: 8-11.

15 Obsérvese que Lindsay en su edición opta por una etimología diferente para estos términos. No es este el lugar de su discusión.

16 Los demás ejemplos son: Brut. 257, parad. 23 y Tusc. III, 77; no hemos registrado ningún uso del derivado verbal en este autor.

17 Cf. Du CANGE (1883: s.v.) y NiERMEYER (1984: s.v.).

18 Los subrayados y la negrita son nuestros.

19 SeRvio, auct. Aen. IX, 707 (cf. Maltby, 1991: s.v.).

20 e.g. Ugutio (cit. Du CANGE, s.v.).

21 CREMASCOLI, 1990: 76. Sobre la influencia que ejerce Isidoro en el periodo medieval conviene consultar AMSLER, 1989: 169-172.

22 CREMASCOLI, 1990: 77.

23 "Singulär ist auch balitor für baliuus, eine weitverbreitete vulgäre Enstellung von baiulus" (PRINZ, 1978: 250).

24 CIL. XIII, 7754 (cf. De RugGIERO, 1961: s.v.).

25 JCUR VI, 15795 (cf. KAJANTO, 1982: 166).

26 PAVIS D'EsCURAC, 1976: 230. 